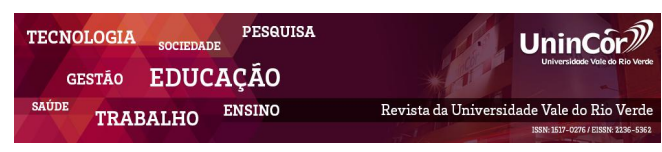

Revista da Universidade Vale do Rio Verde ISSN: 1517-0276 / EISSN: 2236-5362 v. 17 | n. 2 | Ano 2019

Daniel Knebel Baggio

Universidade Regional do Noroeste do Estado do Rio Grande do Sul - UNIJUí

baggiod@unijui.edu.br

Isoé Nícolas Schneider

Universidade Regional do Noroeste do Estado do

Rio Grande do Sul - UNIJUí

iso.nicolas@hotmail.com

Maria Margarete Baccin Brizolla

Universidade Regional do Noroeste do Estado do

Rio Grande do Sul - UNIJUí

marga.brizolla@unijui.edu.br

Argemiro Luis Brum

Universidade Regional do Noroeste do Estado do

Rio Grande do Sul - UNIJUÍ

argelbrum@unijui.edu.br

João Serafim Tusi da Silveira

Universidade Regional Integrada do Alto Uruguai e das Missões/URI

joaotusi@hotmail.com

Eduardo Luiz Goulart Knebel

Universidade Regional do Noroeste do Estado do Rio Grande do Sul - UNIJUí eduardoknebe198@gmail.com

\section{PERSISTÊNCIA DE FUNDOS DE INVESTIMENTOS DE DIREITO \\ CREDITÓRIO DO AGRONEGÓCIO, INDÚSTRIA E COMÉRCIO}

\section{RESUMO}

O objetivo deste artigo foi de analisar a persistência da rentabilidade, do risco e a relação entre estas duas variáveis, dos fundos de investimento em direito creditório do agronegócio, indústria e comércio. Para tanto, os fundos foram ranqueados a partir das variáveis e classificados de acordo com a superioridade ou não da mediana. Para transformar os resultados em medidas estatísticas utilizou-se os modelos propostos por Malkiel (1995), Brown e Goetzmann (1995) e Khan e Rudd (1995). Os resultados encontrados indicam que há persistência em relação à rentabilidade e ao risco. Ou seja, os fundos que possuem maior rentabilidade em um determinado ano, continuam obtendo rentabilidade maior no ano seguinte. Da mesma forma ocorre com o risco. Os fundos com maior risco em um ano, possuem maior risco no ano seguinte. Porém, existe uma relação inversa entre essas variáveis, ou seja, os fundos que possuem rentabilidade maior, são aqueles que tem um risco menor.

Palavras-chave: Persistência. Rentabilidade. Risco. Fundos de Investimento.

\section{PERSISTENCE OF INVESTMENT FUNDS IN CREDITORIAL LAW, AGRIBUSINESS, INDUSTRY AND COMMERCE}

\begin{abstract}
The objective of this article was to analyze the persistence of profitability, risk and the relationship between these two variables, of investment funds in credit law of agribusiness, industry and commerce. To do so, the funds were ranked from the variables and classified according to whether or not the median was superior. In order to transform the results into statistical measures, the models proposed by Malkiel (1995), Brown and Goetzmann (1995) and Khan and Rudd (1995) were used. The results indicate that there is persistence regarding profitability and risk. That is, the funds that have the highest profitability in a given year, continue to obtain higher profitability the following year. Likewise with risk. The funds with the highest risk in one year are at greater risk in the following year. However, there is an inverse relationship between these variables, that is, the funds that have higher profitability, are those that have a lower risk
\end{abstract}

Keywords: Persistence. Profitability. Risk. Investment Funds. 


\section{INTRODUÇÃO}

Uma das opções de investimento, que mais se destaca no mercado financeiro atualmente, é a dos fundos de investimento, tanto pelo crescimento da quantidade e opções oferecidas, quanto pela sua importância na geração de poupança interna e no direcionamento dos recursos em atividades produtivas (Amaral, Vilaça, Barbosa, \& Bressan, 2004). Eles são determinantes para o desenvolvimento econômico das regiões onde os recursos são aplicados (Baggio, 2012).

Fonseca, Bressan, Iquiapaza, \& Guerra (2007) defendem que os fundos permitem que os pequenos investidores tenham acesso a melhores opções de investimento no mercado financeiro, com custos reduzidos, contando com o suporte de uma administração profissional (gestores dos fundos) e a diversificação dos investimentos, que favorecem a diluição do risco e o aumento do potencial de retorno.

De acordo com o Anuário da Indústria de Fundos de Investimento (Fundação Getúlio Vargas [FGV], 2017), o mercado brasileiro de fundos é o décimo maior do mundo, com participação de aproximadamente $3 \%$ do total do patrimônio líquido mundial. Atualmente, mais de 15.000 fundos estão sob gestão no Brasil, se destacando também pela variedade de produtos oferecidos e pela diversidade de investidores (Associação Brasileira das Entidades dos Mercados Financeiro e de Capitais [ANBIMA], 2017).

$\mathrm{Na}$ avaliação do comportamento de um fundo de investimento, devem ser consideradas a sua rentabilidade, o seu risco e a sua liquidez. Em mercados suficientemente líquidos, a relação entre rentabilidade e risco deve ser analisada, levando em consideração as diversas medidas de performance existentes e, também, a eficiência dos gestores dos fundos em comparação com os demais e com o mercado (Baggio, Silveira, Schneider, Maciel, \& Oliva, 2018).

Como explica Vargas (2006), para medir a eficiência na gestão de carteiras, pode-se proceder de duas maneiras. Por um lado, estimando o rendimento médio histórico das carteiras durante um determinado período de tempo para, posteriormente, ajustar estes rendimentos pelo comportamento do mercado de referência e por uma medida representativa de risco, apoiada pela respectiva carteira.

Para tanto, o objetivo principal do presente estudo reside em verificar a persistência das variáveis rentabilidade e risco dos Fundos de Investimentos em Direitos Creditórios (FIDC), Agronegócio, Indústria e Comércio, no período de 2011 a 2016 e a relação existente entre as duas variáveis.

Andaku e Pinto (2003), examinaram o desempenho de 84 fundos mútuos brasileiros no período de julho de 1994 a junho de 2001. E chegam expõem a existência da performance no mercado brasileiro apenas para investimentos de longo prazo. Grinblatt e Titman (1992) encontram resultados semelhante no mercado americano.

\section{METODOLOGIA}

Trata-se de um estudo de natureza quantitativa que, segundo Sampieri et al. (2006) usa coleta de dados, teste de hipóteses, medição numérica e análise estatística. 
Para a composição do conjunto de fundos pesquisados, considerou-se inicialmente todos os fundos de investimento da categoria Fundos de Investimentos em Direitos Creditórios (FIDC) agronegócio, indústria e comércio da ANBIMA, composta por 223 fundos existentes no período 2011-2016. Dentre esses, selecionou-se todos os que estiveram ativos durante os anos de 2011 a 2016.

Para calcular a variável rentabilidade anual dos fundos, foram utilizados os dados dos retornos mensais dos fundos no período de análise, obtendo-se assim um média mensal dos fundos para cada ano analisado. A partir das rentabilidades mensais, realizou-se o desvio padrão das mesmas, obtendo assim a medida de risco de cada fundo em cada ano.

Seguidamente os fundos foram ordenados (ranqueados) a partir da variável rentabilidade $\mathrm{e}$ risco. Os fundos que obtiveram rentabilidade superior a mediana de rentabilidade foram classificados como "Winner" (W), ou seja, ganhadores em rentabilidade. Os Fundos que obtiveram rentabilidade inferior a mediana de rentabilidade foram classificados como "Losers" (L), isto é, perdedores em rentabilidade. O mesmo ocorreu com a variável risco, onde os fundos que obtiveram o maior risco foram classificados como "Winners" e os com menor risco como "Losers".

Logo, para aplicar a metodologia da persistência e verificar se o desempenho dos fundos persiste ao longo dos anos, eles foram analisados de forma pareada, ou seja, se em dois anos consecutivos um fundo obteve a variável superior a mediana da amostra. Caso esta situação tenha ocorrido, o presente fundo foi classificado como "Winner, Winner" (WW). Já os fundos que obtiveram variável superior no primeiro ano e inferior que a mediana no ano seguinte foram classificadas como "Winner, Loser" (W, L). Já os fundos que obtiveram performance inferior no primeiro ano e superior a mediana no segundo ano foram classificadas como "Loser, Winner" (L, W). Por fim, aqueles fundos que obtiveram performance inferior que a mediana no primeiro ano e no ano seguinte foram classificadas como "Loser, Loser" (L, L).

Os fundos foram analisados em três momentos: 1) a partir das suas rentabilidades, para verificar se o desempenho de um gestor persiste ao longo do tempo; 2) pelos seus riscos; 3) relação risco e retorno, para observar se os fundos que obtiveram os melhores desempenhos foram os quais obtiveram os mais elevados riscos.

Por fim, para transformar os resultados em medidas estatísticas de análise, utilizou-se dos modelos propostos por Malkiel (1995), Brown e Goetzmann (1995) e Khan e Rudd (1995). Estes replicados por Baggio e Agudo (2008), Baggio et al (2014) e Agudo, Baggio e Compains (2017). Malkiel (1995) propõe a prova Z pela expressão:

$$
Z=(y-n p) / \sqrt{n p(1-p)}
$$

Onde, z é a variável estatística que segue uma distribuição normal $(0,1)$, y é o número de fundos ganhadores em dois períodos consecutivos, $\mathrm{n}=$ WW (fundos ganhadores na variável de análise) $+\mathrm{y}$ (fundos que passaram de ganhadores para perdedores) e o $\mathrm{p}=0,5$

A proporção (rating) de disparidade RD aplicado pelo método Brown e Goetzmann(1995) é: 


$$
\mathrm{RD}=\frac{W W \times L L}{W L \times L W}
$$

Onde $\mathrm{WW}=$ fundos que obtiveram a variável $i$ superior a mediana em dois períodos consecutivos, $\mathrm{LL}=$ fundos que obtiveram a variável $i$ inferior em dois períodos consecutivos, $\mathrm{WL}=$ fundos que obtiveram a variável $i$ superior em um primeiro momento e inferior no segundo e $\mathrm{LW}=$ fundos que obtiveram a variável $i$ inferior no primeiro período e superior no segundo.

A partir de RD pode-se calcular um novo valor de Z, com distribuição normal $(0,1)$ :

$$
Z=\frac{\ln (R D)}{\sigma \ln (R D)}
$$

Em que:

$$
\sigma_{\log (R D)}=\sqrt{1 / W W+1 / W L+1 / L W+1 / L L}
$$

Pelo Metodo $\mathrm{x}^{2}$ de Khan e Rudd (1995) a expressão é a seguinte:

$$
\chi^{2}=\sum_{i=1}^{n} \sum_{j=1}^{n} \frac{\left(O_{i j}-E_{i j}\right)^{2}}{E_{i j}}
$$

Onde $\mathrm{O} i j=$ Frequência encontrada na célula de interseção da linha $i$ com a coluna $j$ e E $i j=$ Frequência esperada na célula de interseção da linha $i$ com a coluna $j$. Portanto, a fórmula de $\mathrm{x}^{2}$ de Kahn e Rudd (1995) é a seguinte:

$$
x^{2}=\frac{(W W-N / 4)^{2}}{N / 4}+\frac{(W L-N / 4)^{2}}{N / 4}+\frac{(L W-N / 4)^{2}}{N / 4}+\frac{(L L-N / 4)^{2}}{N / 4}
$$

Onde, $\mathrm{N}$ é o número total de indicadores analisados. Para o caso de uma matriz $(2 \times 2)$ esta fórmula apresenta um grau de liberdade. As quatro células da matriz têm uma frequência idêntica: número de indicadores dividido por quatro.

A utilização destas provas estatísticas permite verificar a seguinte hipótese: Hipótese: $H 0=$ Não existe relação significativa entre a variável $i$ passada e variável $i$ futura dos fundos FIDC, agronegócio, indústria e comércio. $H a=$ Existe relação significativa entre a variável $i$ passada $\mathrm{e}$ futura dos fundos FIDC, agronegócio, indústria e comércio. $\mathrm{O}$ valor $p$ quando inferior a 5\% permite rechaçar a hipótese $H O$ aceitando a hipótese $H a$.

\section{RESULTADOS}

\subsection{Rentabilidade}

Em relação a análise de persistência da rentabilidade, no quadro 01, pode-se perceber uma concentração de fundos nas extremidades (WW e LL), confirmando assim a persistência da rentabilidade no período analisado.

Quadro 01 - Resumo persistência dos fundos

\begin{tabular}{|l|l|l|l|l|l|l|}
\hline & $\mathbf{2 0 1 1}$ & $\mathbf{2 0 1 2}$ & $\mathbf{2 0 1 3}$ & $\mathbf{2 0 1 4}$ & $\mathbf{2 0 1 5}$ & \multirow{2}{*}{ TOTAL } \\
\hline & $\mathbf{2 0 1 2}$ & $\mathbf{2 0 1 3}$ & $\mathbf{2 0 1 4}$ & $\mathbf{2 0 1 5}$ & $\mathbf{2 0 1 6}$ & \multirow{2}{*}{ WW } \\
\hline WL & 30 & 31 & 30 & 31 & 27 & 149 \\
\hline LW & 9 & 11 & 8 & 7 & 10 & 45 \\
\hline LL & 9 & 10 & 8 & 7 & 10 & 44 \\
\hline $\mathbf{N}^{\mathbf{0}}$ FUNDOS & 70 & 30 & 31 & 31 & 28 & 150 \\
\hline
\end{tabular}

$* \mathrm{WW}=$ Fundos que obtiveram rentabilidade superior em $t$ e em $t_{+1}$. $\mathrm{LL}=$ Fundos que obtiveram rentabilidade inferior em $t$ e em $\mathrm{t}_{+1} . \mathrm{WL}=$ Fundos que obtiveram rentabilidade superior em $t$ e inferior em $t_{+1}$. $\mathrm{LW}=$ Fundos que obtiveram rentabilidade inferior em $\mathrm{t}$ e superior em $\mathrm{t}_{+1}$. 
Nas medidas de Brown e Goetzmann (1995), Khan e Rudd (1995) e Malkiel (1995), se comprova tal persistência, uma vez que os valores de p são inferiores a 0,5 (quadro 02).

Quadro 02 - Análise da persistência da rentabilidade

\begin{tabular}{|c|c|c|c|c|c|c|}
\hline & $\mathbf{2 0 1 1}$ & $\mathbf{2 0 1 2}$ & $\mathbf{2 0 1 3}$ & $\mathbf{2 0 1 4}$ & $\mathbf{2 0 1 5}$ & \multirow{2}{*}{ TOTAL } \\
\hline & $\mathbf{2 0 1 2}$ & $\mathbf{2 0 1 3}$ & $\mathbf{2 0 1 4}$ & $\mathbf{2 0 1 5}$ & $\mathbf{2 0 1 6}$ & \multirow{2}{*}{ TO14 } \\
\hline $\mathbf{Z}$ & 3,363 & 3,086 & 3,569 & 3,893 & 2,795 & 7,4668 \\
\hline $\mathbf{P}$ & $\mathbf{0 , 0 0 1}$ & $\mathbf{0 , 0 0 2}$ & $\mathbf{0 , 0 0 0}$ & $\mathbf{0 , 0 0 0}$ & $\mathbf{0 , 0 0 5}$ & $\mathbf{0 , 0 0 0 0}$ \\
\hline OR & 11,111 & 8,455 & 14,531 & 19,612 & 7,560 & 11,2879 \\
\hline & 0,5375 & 0,5065 & 0,5618 & 0,5918 & 0,5223 & 0,2415 \\
\hline In (OR) & 2,4079 & 2,1347 & 2,6763 & 2,9762 & 2,0229 & 2,4237 \\
\hline $\mathbf{Z}$ & 4,4800 & 4,2150 & 4,7640 & 5,0290 & 3,8733 & 10,0357 \\
\hline $\mathbf{P}$ & $\mathbf{0 , 0 0 0}$ & $\mathbf{0 , 0 0 0}$ & $\mathbf{0 , 0 0 0}$ & $\mathbf{0 , 0 0 0}$ & $\mathbf{0 , 0 0 0}$ & $\mathbf{0 , 0 0 0 0}$ \\
\hline $\mathbf{P}$ & 22,615 & 19,561 & 26,325 & 30,316 & 16,360 & 282,2996 \\
\hline & $\mathbf{0 , 0 0 0}$ & $\mathbf{0 , 0 0 0}$ & $\mathbf{0 , 0 0 0}$ & $\mathbf{0 , 0 0 0}$ & $\mathbf{0 , 0 0 0}$ & $\mathbf{0 , 0 0 0 0}$ \\
\hline
\end{tabular}

*Estatisticamente significativo a um nível de 1\%. $Z^{1}$ : Representa o Z-test do modelo de Malkiel (1995); OR: Expressa o índice proposto por Brown e Goetzmann (1995); $Z^{2}$ : a partir do ID de Brown e Goetzmann se propõem o novo $Z$-valor, com uma distribuição normal $(0,1) ; \chi^{2}$ :Test da $\chi^{2}$ de Kahn e Rudd (1995).

\subsection{Risco}

Da mesma forma que na análise sobre a rentabilidade, a análise do risco nos mostra concentração dos fundos nas extremidades, confirmando assim a hipótese de que há persistência no risco dos fundos analisados (quadro 03).

Quadro 03 - Resumo persistência do risco

\begin{tabular}{|c|c|c|c|c|c|c|}
\hline & $\mathbf{2 0 1 1}$ & $\mathbf{2 0 1 2}$ & $\mathbf{2 0 1 3}$ & $\mathbf{2 0 1 4}$ & $\mathbf{2 0 1 5}$ & \multirow{2}{*}{ TOTAL } \\
\hline WW & $\mathbf{2 0 1 2}$ & $\mathbf{2 0 1 3}$ & $\mathbf{2 0 1 4}$ & $\mathbf{2 0 1 5}$ & $\mathbf{2 0 1 6}$ & TO \\
\hline WL & 90 & 30 & 30 & 29 & 29 & 148 \\
\hline LW & 9 & 12 & 8 & 8 & 8 & 45 \\
\hline LL & 30 & 30 & 31 & 30 & 30 & 151 \\
\hline N $^{\mathbf{0}}$ FUNDOS & 78 & 84 & 77 & 75 & 75 & 239 \\
\hline
\end{tabular}

* $\mathrm{WW}=$ Ações que obtiveram risco superior em $t$ e em $t+1 . \mathrm{LL}=$ Ações que obtiveram risco inferior em $t$ e em $\mathrm{t}_{+l}$. WL= Ações que obtiveram risco superior em $t \mathrm{e}$ inferior em $\mathrm{t}+1$. $\mathrm{LW}=$ Ações que obtiveram risco inferior em $\mathrm{t}$ e superior em $\mathrm{t}_{+1}$.

Para comprovar estatisticamente, foram novamente utilizados os testes propostos por Brown e Goetzmann (1995), Khan e Rudd (1995) e Malkiel (1995), tais testes comprovam a persistência uma vez que o valor de $p$ em todos os testes, se manteve abaixo de 0,5 (quadro 04 ). 
Quadro 04 - Análise da persistência do risco

\begin{tabular}{|c|c|c|c|c|c|c|}
\hline & $\mathbf{2 0 1 1}$ & $\mathbf{2 0 1 2}$ & $\mathbf{2 0 1 3}$ & $\mathbf{2 0 1 4}$ & $\mathbf{2 0 1 5}$ & \multirow{2}{*}{} \\
\cline { 1 - 6 } & $\mathbf{2 0 1 2}$ & $\mathbf{2 0 1 3}$ & $\mathbf{2 0 1 4}$ & $\mathbf{2 0 1 5}$ & $\mathbf{2 0 1 6}$ & \multirow{2}{*}{ TOTAL } \\
\hline $\mathbf{Z}$ & 3,363 & 2,777 & 3,569 & 3,452 & 3,452 & 7,4141 \\
\hline $\mathbf{P}$ & $\mathbf{0 , 0 0 1}$ & $\mathbf{0 , 0 0 5}$ & $\mathbf{0 , 0 0 0}$ & $\mathbf{0 , 0 0 1}$ & $\mathbf{0 , 0 0 1}$ & $\mathbf{0 , 0 0 0 0}$ \\
\hline $\mathbf{O R}$ & 11,111 & 6,250 & 14,531 & 13,594 & 13,594 & 11,0360 \\
\hline & 0,5375 & 0,4830 & 0,5618 & 0,5638 & 0,5638 & 0,2405 \\
\hline In (OR) & 2,4079 & 1,8326 & 2,6763 & 2,6096 & 2,6096 & 2,4012 \\
\hline $\mathbf{Z}$ & 4,4800 & 3,7938 & 4,7640 & 4,6290 & 4,6290 & 9,9855 \\
\hline $\mathbf{P}$ & $\mathbf{0 , 0 0 0}$ & $\mathbf{0 , 0 0 0}$ & $\mathbf{0 , 0 0 0}$ & $\mathbf{0 , 0 0 0}$ & $\mathbf{0 , 0 0 0}$ & $\mathbf{0 , 0 0 0 0}$ \\
\hline & 22,615 & 15,429 & 26,325 & 24,680 & 24,680 & 276,9833 \\
\hline $\mathbf{P}$ & $\mathbf{0 , 0 0 0}$ & $\mathbf{0 , 0 0 0}$ & $\mathbf{0 , 0 0 0}$ & $\mathbf{0 , 0 0 0}$ & $\mathbf{0 , 0 0 0}$ & $\mathbf{0 , 0 0 0 0}$ \\
\hline
\end{tabular}

*Estatisticamente significativo a um nível de $1 \%$. $Z^{1}$ : Representa o Z-test do modelo de Malkiel (1995); OR: Expressa o índice proposto por Brown e Goetzmann (1995); $Z^{2}$ : a partir do ID de Brown e Goetzmann se propõem o novo Z-valor, com uma distribuição normal $(0,1) ; \chi^{2}$ :Test da $\chi^{2}$ de Kahn e Rudd (1995).

\subsection{Rentabilidade e Risco}

Em relação à rentabilidade e risco (quadro 05), percebe-se uma relação inversa, uma vez que os fundos se concentraram no meio da tabela (WL e LW), isso demonstra que os fundos que são ganhadores em risco (possuem maior risco) são os que tem menor rentabilidade (perdedores em rentabilidade). E os que possuem maior rentabilidade, são aqueles que tem um menor risco.

Quadro 05 - Resumo persistência da rentabilidade e risco

\begin{tabular}{|c|c|c|c|c|c|c|c|}
\hline & $\mathbf{2 0 1 1}$ & $\mathbf{2 0 1 2}$ & $\mathbf{2 0 1 3}$ & $\mathbf{2 0 1 4}$ & $\mathbf{2 0 1 5}$ & $\mathbf{2 0 1 6}$ & \multirow{2}{*}{} \\
\hline & $\mathbf{2 0 1 1}$ & $\mathbf{2 0 1 2}$ & $\mathbf{2 0 1 3}$ & $\mathbf{2 0 1 4}$ & $\mathbf{2 0 1 5}$ & $\mathbf{2 0 1 6}$ & \multirow{2}{*}{ TOTAL } \\
\hline WW & 17 & 22 & 17 & 18 & 13 & 18 & 105 \\
\hline WL & 37 & 33 & 33 & 32 & 36 & 29 & 200 \\
\hline LW & 37 & 33 & 33 & 32 & 36 & 29 & 200 \\
\hline LL & 18 & 22 & 18 & 19 & 13 & 19 & 109 \\
\hline N $^{\mathbf{0}}$ FUNDOS & 109 & 110 & 101 & 101 & 98 & 95 & 320 \\
\hline
\end{tabular}

* $\mathrm{WW}=$ Ações que obtiveram rentabilidade e risco superior em $t$. LL= Ações que obtiveram rentabilidade e risco inferior em $t . \mathrm{WL}=$ Ações que obtiveram rentabilidade superior e risco inferior em $\mathrm{t}$. $\mathrm{LW}=$ Ações que obtiveram rentabilidade inferior e risco superior em t.

Para comprovar essa relação inversa, foram utilizados os testes estatísticos de Brown Goetzmann (1995), Khan e Rudd (1995) e
Malkiel (1995), sendo que os mesmos, possuem valor de $p$ menor que 0,5 , (quadro 06) confirmando o que foi exposto no parágrafo anterior. 
Quadro 06 - Análise da persistência da rentabilidade e risco

\begin{tabular}{|c|c|c|c|c|c|c|c|}
\hline & $\mathbf{2 0 1 1}$ & $\mathbf{2 0 1 2}$ & $\mathbf{2 0 1 3}$ & $\mathbf{2 0 1 4}$ & $\mathbf{2 0 1 5}$ & $\mathbf{2 0 1 6}$ & \multirow{2}{*}{ TOTAL } \\
\hline & $\mathbf{2 0 1 1}$ & $\mathbf{2 0 1 2}$ & $\mathbf{2 0 1 3}$ & $\mathbf{2 0 1 4}$ & $\mathbf{2 0 1 5}$ & $\mathbf{2 0 1 6}$ & \multirow{2}{*}{ TOT } \\
\hline $\mathbf{Z}$ & $-2,722$ & $-1,483$ & $-2,263$ & $-1,980$ & $-3,286$ & $-1,605$ & $-5,4397$ \\
\hline $\mathbf{p}$ & $\mathbf{0 , 0 0 6}$ & $\mathbf{0 , 1 3 8}$ & $\mathbf{0 , 0 2 4}$ & $\mathbf{0 , 0 4 8}$ & $\mathbf{0 , 0 0 1}$ & $\mathbf{0 , 1 0 9}$ & $\mathbf{0 , 0 0 0 0}$ \\
\hline $\mathbf{O R}$ & 0,224 & 0,444 & 0,281 & 0,334 & 0,130 & 0,407 & 0,2861 \\
\hline & 0,4104 & 0,3892 & 0,4183 & 0,4131 & 0,4576 & 0,4209 & 0,1694 \\
\hline $\mathbf{I n}(\mathbf{O R})$ & $-1,4983$ & $-0,8109$ & $-1,2694$ & $-1,0967$ & $-2,0371$ & $-0,8998$ & $-1,2513$ \\
\hline $\mathbf{Z}$ & $-3,6507$ & $-2,0833$ & $-3,0346$ & $-2,6544$ & $-4,4517$ & $-2,1378$ & $-7,3866$ \\
\hline $\mathbf{p}$ & $\mathbf{0 , 0 0 0}$ & $\mathbf{0 , 0 3 7}$ & $\mathbf{0 , 0 0 2}$ & $\mathbf{0 , 0 0 8}$ & $\mathbf{0 , 0 0 0}$ & $\mathbf{0 , 0 3 3}$ & $\mathbf{0 , 0 0 0 0}$ \\
\hline & 13,972 & 4,400 & 9,535 & 7,238 & 21,592 & 4,663 & 378,3250 \\
\hline $\mathbf{p}$ & $\mathbf{0 , 0 0 0}$ & $\mathbf{0 , 0 3 6}$ & $\mathbf{0 , 0 0 2}$ & $\mathbf{0 , 0 0 7}$ & $\mathbf{0 , 0 0 0}$ & $\mathbf{0 , 0 3 1}$ & $\mathbf{0 , 0 0 0 0}$ \\
\hline
\end{tabular}

*Estatisticamente significativo a um nível de $1 \%$. $Z^{1}$ : Representa o Z-test do modelo de Malkiel (1995); OR: Expressa o índice proposto por Brown e Goetzmann (1995); Z $Z^{2}$ : a partir do ID de Brown e Goetzmann se propõem o novo $Z$-valor, com uma distribuição normal $(0,1) ; \chi^{2}$ :Test da $\chi^{2}$ de Kahn e Rudd (1995).

\section{CONCLUSÕES}

Ao analisar os fundos FIDC, Agro e Comércio pode-se perceber a persistência da rentabilidade destas opções de investimento, sendo que durante o período de análise, a grande maioria dos fundos se manteve como ganhadores (WW) ou perdedores (LL) e nas medidas propostas por Goetzmann (1995), Khan e Rudd (1995) e Malkiel (1995) confirma-se a persistência da rentabilidade.

Da mesma forma que pode-se visualizar a persistência da rentabilidade dos fundos FIDC, Agro e Comércio, pode-se perceber também a persistência do risco dessas opções de investimento, sendo que igualmente a rentabilidade, a grande maioria dos fundos se mantém nas extremidades (WW e LL), confirmados pela metodologia de Goetzmann (1995), Khan e Rudd (1995) e Malkiel (1995).

Quando analisado a relação entre risco e rentabilidade, a concentração dos fundos se deu no meio da tabela, obtendo-se assim uma relação inversa na maioria dos fundos. Ou seja, diferindo da ideia teórica de que quanto maior o risco, maior a rentabilidade, nesse caso quanto maior o risco, menor a rentabilidade e vice-versa (quanto menor o risco, maior a rentabilidade).

\section{REFERÊNCIAS}

AGUDO, L. F.; BAGGIO, D. K.; COMPAINS, F. J. R. Las medidas de eficiencia en la gestión: un estudio de los fondos de inversión socialmente responsables (fisr) de Brasil. Revista Gesto, v.5, p.74 - 92, 2017.

AMARAL, H. F., VILAÇA, C. S. I., BARBOSA, C. F. M., \& BRESSAN, V. G. F. Fundos de pensão como financiadores da atividade econômica. Revista de Administração de Empresas, São Paulo, 44 (2), 7991, Junho, 2004.

ANDAKU, F. T. A.; PINTO, A. C. F. A persistência de desempenho dos fundos de investimento em ações no Brasil. Revista de Economia e Administração. v. 2; p. 23-33, abr-jun, 2003.

ASSOCIAÇÃO BRASILEIRA DAS ENTIDADES DOS MERCADOS FINANCEIRO E DE CAPITAIS. (2017) Consolidado Histórico de Fundos de Investimento Julho/2017. Disponível em $<$ http://www.anbima.com.br/pt_br/informar/estatistica s/fundos-de-investimento/fi-consolidadohistorico.htm> Acesso em: 01. Out. 2018.

BAGGIO, D. K. Los fondos de inversión socialmente responsable de Brasil. 2012. Tese (Doutorado em Contabilidade e Finanças) - Faculdade de Economia e Empresas, Universidad de Zaragoza. Zaragoza, 2012.

BAGGIO, D. K., SILVEIRA, J. S. T., SCHNEIDER, I. N., MACIEL, A. C., \& OLIVA, E. C. Las medidas de performance en la gestión de fondos de inversión 
socialmente responsables. Revista Academica

Contribuciones a la Economia. abril - junho, 2018.

BAGGIO, D. K.; AGUDO, L. F. Es la performance de los Fondos Brasileños de Renta Variable Persistente?.

Gestión Joven - Revista de la Agrupación Joven Iberoamericana de Contabilidad y Administración de Empresas. v.1, p.01, 2008.

BAGGIO, D. K.; KELM, M.; ZANELLA, W.; MACIEL, A. C.; SILVA, A. J.

Análisis de la persistencia en el desempeño de los fondos de inversión de renta variable de brasil. Revista de Contabilidade, Ciência da Gestão e Finanças. v.2, p.26 - 40, 2014.

BROWN, S. y GOETZMANN, W. N. "Performance persistence". Journal of Finance, Junio. 1995.

FONSECA, N. F., BRESSAN, A. A., IQUIAPAZA, R. A., \& GUERRA, J. P. Análise do Desempenho Recente de Fundos de Investimento no Brasil”.

Revista de Contabilidade Vista, 18(1), 95-116, 2007.

FUNDAÇÃO GETÚLIO VARGAS. Anuário da Indústria de Fundos de Investimento. São Paulo: FGV, 2017.

GRINBLATT, M; TITMAN, S. The persistence of mutual fund performance. The Journal of Finances, v. 47, p. 1977-1984, 1992.

KAHN, R.N.; RUDD, A. Does historical performance predict future performance? Financial Analysts Journal, v.51, p.43-52, 1995.

MALKIEL, B. "Returns from investing in equity mutual funds 1971 to 1991 ". Journal of Finance, v. 50, p. 549-572, 1995.

SAMPIERI, R. H.; COLLADO, C. F; LUCIO, P. B. Metodologia de pesquisa. 3 ed. São Paulo: McGraw Hill, 2006.

SHARPE, W. F. Capital asset prices: A theory of market equilibrium under conditions of risk. The Journal of Finance, v. 19, n. 3, p. 425-442, Set, 1964.

SHARPE, W.F. "Mutual fund performance”, Journal of Business, v.39, p.119-138, 1966.

VARGAS, M. M. Fondos de Inversión españoles: Análisis empírico de eficiencia y persistencia en la gestión. 2006. Tesis (Doutorado em Contabilidade e Finanças) - Faculdade de Economia e Empresas. Universidad de Zaragoza. Zaragoza, 2006.

\footnotetext{
Daniel Knebel Baggio

Possui graduação em Administração pela Universidade Regional do Noroeste do Estado do Rio Grande do Sul (UNIJUI - 2005), Mestrado em Contabilidade e Finanças - Universidad de Zaragoza
}

(2007) e Doutorado em Contabilidade e Finanças Universidad de Zaragoza (2012) revalidado pela Universidade de São Paulo (USP) em Controladoria e Contabilidade. Atualmente é professor do Programa de Pós-Graduação em Desenvolvimento Regional da UNIJUI e do Programa de PósGraduação em Gestão Estratégica de Organizações da Universidade Regional Integrada (URI). Investigador pela Universidade de Zaragoza, integrando o Grupo de Investigación en Economia Financiera (GIECOFIN), da UNIJUI, integrando o Grupo de Pesquisa em Competitividade e Gestão Estratégica para o Desenvolvimento (GPCOM) e da URI, integrando o Grupo de Pesquisa de Métodos Aplicados à Gestão Organizacional (MAGO). Tem experiência na área de Administração, com ênfase em Administração Financeira, atuando principalmente nos seguintes temas: Finanças, Mercado de Capitais, Fundos e Análise de Investimentos, Governança Corporativa, Empreendedorismo, Jogos Empresariais e Métodos Quantitativos de Pesquisa.

\begin{abstract}
Isoé Nícolas Schneider
Possui graduação em Administração pela Universidade Regional do Noroeste do Estado do Rio Grande do Sul (2016). Aperfeiçoamento em Finanças e Mercado de Capitais pela Universidade Regional do Noroeste do Estado do Rio Grande do Sul (2018). Mestrando em Desenvolvimento Regional pela Universidade Regional do Noroeste do Estado do Rio Grande do Sul. Investigador pela Universidade Regional do Noroeste do Estado do Rio Grande do Sul, integrando o Grupo de Pesquisa em Competitividade e Gestão Estratégica para o Desenvolvimento (GPCOM) e pela Universidade Regional Integrada, integrando o Grupo de Pesquisa de Métodos Aplicados à Gestão Organizacional (MAGO) Tem experiência na área de Administração, com ênfase em Administração Financeira, Mercado de Capitais, Fundo de Investimentos, Governança Corporativa, Responsabilidade Social Empresarial
\end{abstract}

\begin{abstract}
Maria Margarete Baccin Brizolla
Possui graduação em Ciências Contábeis e Administração pela UNIJUI/RS (1996; 2013), mestre em Ciências Contábeis pela UNISINOS/RS (2004). Doutora em Ciências Contábeis e Administração pela FURB/SC (2016) e Pós Doutorado em Contabilidade na UFSC/SC (2018). Atualmente é professora da UNIJUI, Tem experiência em Contabilidade com ênfase em gestão de empresas, atuando principalmente na contabilidade geral, contabilidade e gestão ambiental, sustentabilidade, gestão financeira, análise de viabilidade e custos.
\end{abstract}

\footnotetext{
Argemiro Luis Brum

Possui graduação em Administração de Empresas pela Universidade Regional do Noroeste do Estado do Rio Grande do Sul (1981), graduação em
} 
Tecnólogo Em Cooperativismo pela Universidade Regional do Noroeste do Estado do Rio Grande do Sul (1981), mestrado em Economia Agrícola Institut Agronomique Méditerranéen de Montpellier (1986) e doutorado em Economia Internacional Ecole des Hautes Etudes en Sciences Sociales (1989). Atualmente é professor titular da Universidade Regional do Noroeste do Estado do Rio Grande do Sul, consultor sênior do SEBRAE, consultor de empresas nacionais e internacionais na área do agronegócio, coordenador da Central Internacional de Análises Econômicas e de Estudos de Mercado Agropecuário (CEEMA/DACEC/UNIJUI), membro do Colegiado de Coordenação do Mestrado em Desenvolvimento (UNIJUI). Tem experiência na área de Economia, com ênfase em Relações do Comércio; Política Comercial; Integração Econômica e Mercados Agropecuários, atuando principalmente nos seguintes temas: economia, economia internacional, negociações comerciais multilaterais, mercados, soja, grãos em geral e produção. vinculado ao Programa. Seu principal interesse em pesquisa relaciona-se a Métodos Quantitativos aplicados às Ciências Sociais e às Engenharias, em contextos de Gestão Organizacional e de Desenvolvimento Local e Regional Sustentável.

Eduardo Luiz Goulart Knebel

Graduando do curso de Agronomia da UNIJUí

\begin{abstract}
João Serafim Tusi da Silveira
Possui graduação em Economia pela Universidade Federal de Santa Maria (UFSM), RS, Brasil (1972) e mestrado (1976) e doutorado (2000) em Engenharia de Produção pela Universidade Federal de Santa Catarina (UFSC), SC, Brasil. Foi Professor do Depto. de Ciências Econômicas e do PPG em Eng ${ }^{\mathrm{a}}$ de Produção da UFSC (1975-2003), onde é membro fundador da Fundação de Estudos e Pesquisas SócioEconômicas (FEPESE). Orientou 18 dissertações de MS, integrou 62 bancas de MS e 16 de DR, e publicou 161 trabalhos até o momento. Foi, também, Economista Chefe da Assessoria de Planejamento da Fundação de Amparo ao Meio Ambiente (FATMA/SC); Subsecretário de Estudos Geográficos e Estatísticos da Secretaria de Estado de Coordenação Geral e Planejamento (SEPLAN/SC); e Consultor do PNUD - Programa das Nações Unidas para o Desenvolvimento. Com relação a títulos e prêmios, destacou-se em 2012 como Membro Fundador do EURO Working Group on Stochastic Programming and Applications; em 2010, conquistou o $2^{\circ}$ lugar, no $1^{\circ}$ Prêmio Nacional de Desenvolvimento Regional - homenagem a Celso Furtado, Ministério da Integração Nacional; em 2005, o $1^{\circ}$ Lugar no $1^{\circ}$ Prêmio FGTS Celso Furtado, Ministério do Trabalho e Emprego / CNT / CEF; e, também, neste mesmo ano, Menção Honrosa no $1^{\circ}$ Prêmio Nacional em Economia da Saúde, Ministério da Saúde / IPEA; e, em 2018, Menção Honrosa no $8^{\circ}$ Prêmio Chico Ribeiro - VIII Congresso Internacional de Contabilidade, Custos e Qualidade do Gasto no Setor Público. Atualmente, é professor pesquisador do Programa de Pós-Graduação em Gestão Estratégica de Organizações - PPGGEO - Mestrado Profissional, da Universidade Regional Integrada do Alto Uruguai e das Missões/URI (Campus de Santo Ângelo, RS, Brasil), onde integra o corpo docente e é Editor Chefe da Revista GESTO, periódico semestral
\end{abstract}

